volving $w$ and the $u_{i}$ alone, any algebraically irreducible form which is of the least rank in w. Such a form, however, was found to be a member of $\Omega_{2}$ and hence may be identified with the form $B$.

$B_{1}$ is contained in $\Omega_{1}$. It is reduced with respect to $B$ and, of all such forms in $\Omega_{1}$ in the $u_{i}$, w, and $y_{1}$, it certainly has a lowest rank. Consequently we may replace $A_{1}$, in (1), by $B_{1}$. Continuing, we find that (2) is a basic set for $\Omega_{1}$. Then $\Sigma_{1}$ and $\Sigma_{2}$ are identical. This contradiction proves that $A$ is of lower order in $w$ than $B$ and establishes our theorem.

Columbia University

\title{
AXIOM $C$ OF HAUSDORFF AND THE PROPERTY OF BOREL-LEBESGUE*
}

\section{BY SELBY ROBINSON $\dagger$}

1. Introduction. This is a study in an abstract space $(P, K)$ of the Hausdorff $\ddagger$ property $C$ which may be expressed in the form the interior of every set is an open set. A point $p$ of the space $P$ is interior to a set $V$, if $p$ is a point of $V$ and is not a $K$-point (point of accumulation, limit point) of any subset of $C(V)$. An open set is one all of whose points are interior points. We say that space $(P, K)$ has property $B$ of Hausdorff if and only if any point $p$ which is interior to each of two sets is interior to their logical product; we shall designate as the open set $B$ property, the weaker property: the product of two open sets is an open set. $\S$ By the Hausdorff property $D$ we shall mean that any two points are respectively interior to sets which are disjoined, while in the open set $D$ property the points are required to be in disjoined open sets. The Borel and Borel-Lebesgue properties take three non-equivalent forms in spaces not having property $C$. These three forms coincide if property $C$ is present as do the two forms of property $B$ and of property $D$. In $\$ 3$ we consider three

* Presented to the Society, October 29, 1932.

$\dagger$ National Research Fellow.

$\ddagger$ F. Hausdorff, Grundzüge der Mengenlehre, first edition, 1914, p. 213.

$\S$ Chittenden chose the open set $B$ property as the one to designate as the Hausdorff $B$ property. See Transactions of this Society, vol. 31 (1929), p. 315. 
set functions introduced by Chittenden (loc. cit.) which are analogous, respectively, to the three forms of the Borel and Borel-Lebesgue properties. We show that a necessary and suffcient condition for property $C$ is that some two of these three functions coincide for all sets of the space. In $\S 4$ we prove that a space having properties $B$ and $D$ and a local form of the BorelLebesgue property also has property $C$.

2. The Borel Property. A set $E$ has the Borel property if every enumerable family of sets $F$ which properly covers $E$ (that is, every point of $E$ is interior to some set of the family $F$ ) has a

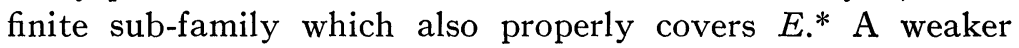
property asserts that any enumerable proper covering $F$ of $E$ has a finite sub-family $F_{1}$ whose sets contain all points of $E$ ( $F_{1}$ covers $E$ simply). By the still weaker open set Borel property, an enumerable family of open sets which covers $E$ is reducible to a finite one. In a euclidean space the sets having these properties are those which are closed and bounded; while the bounded sets are the sets $E$ having the property that every enumerable family of open sets which covers the space $P$ has a finite subfamily which covers $E$. The latter property implies that every infinite sub-set $Q$ of $E$ has a $V$-point $\dagger$ in the space. If the space has property $B$ and no point has another as a limit point, a point which is a $V$-point of an enumerably infinite set is also a nuclear point of that set. But I have shown that a necessary and sufficient condition that every enumerably infinite subset of $E$ have a nuclear point is that every enumerable proper covering of $P$ be reducible to a finite simple covering $\ddagger$ of $E$. Thus the latter property is equivalent to the reducibility of enumerable families of open sets which cover $P$ to finite coverings of $E$, in case property $B$ holds and no point has another as a limit point For the case $E=P$, we formulate this theorem as follows.

Theorem 1. A necessary and sufficient condition that a space $(P, K)$ in which property $B$ holds and no point has another as

* The Borel-Lebesgue property states that proper coverings of any power are reducible to finite ones.

$\dagger$ A point $p$ is a $V$-point of a set $Q$ if for every set $W$ to which $p$ is interior $Q \cdot W$ contains a point other than $p$; a nuclear point if the cardinal number of $Q \cdot W$ is the same as that of $Q$.

$\ddagger$ Covering theorems in general topolngy, American Journal of Mathematics, vol. 55 (1933), Theorem 2. 
limit point, have the open set Borel property is that every enumerable proper covering be reducible to a finite simple one.

In particular, the properties are equivalent in a Fréchet $\mathcal{L}$ space. Chittenden has given an example* of a Fréchet $\mathcal{L}$-space having the properties of Theorem 1 but not the Borel property, and we give another in $\$ 4$. The following example of a space having the open set Borel property but not the "proper to simple" form, shows that the condition, no single point has a limit point, is essential in Theorem 1 . The space consists of a sequence of points $\left\{p_{n}\right\}$ and the sets having $p_{n}$ as a limit point are exactly those which contain $p_{n-1}$.

The following is an example of a set $E$ of a Fréchet $\mathcal{L}$-space which has the open set Borel property but not the "proper to simple" form. The space consists of two concentric circles $D$ and $E$. The sets which have any point $p$ for a limit point are exactly those which ordinarily would have as a limit point the corresponding point $q$ of the other circle. Any enumerable family of sets which are open sets in the space $P=D+E$ has a finite sub-family which covers $E$; but if $E$ is considered as a space in itself, no set has a limit point and the open set Borel theorem is not valid. In contrast to this, it is a stronger condition for a subset $E$ to have the Borel property than for $E$ considered as a space to have the Borel property. For consider a Fréchet $\mathcal{L}$-space $P$ consisting of a plane with the following limit relations. Any point $p$ whose $x$-coordinate is irrational is a limit point of those sets whose intersection with the horizontal line containing $p$ woùld ordinarily have $p$ as a limit point; while a point $p$ whose $x$-coordinate is rational, is a limit point of the sets whose intersection either with the horizontal or the vertical line containing $p$ would ordinarily have $p$ for a limit point. A closed segment $E$ of a horizontal line does not have the Borel property, but does have it if $E$ is considered as a space. Finally in the "proper to simple" case, it makes no difference whether $E$ be considered as a space or as a subset.

3. Set Functions. Analogous to $V(E)$ is the set $O(E)$ of all points $p$ such that every open set containing $p$ contains a point of $E$ other than $p$, and the set $H(E)$ of all points $p$ such that

* This Bulletin, vol. 25 (1918), p. 64. 
every set to which $p$ is interior has in its interior a point of $E$ other than $p$. If we omit the phrase "other than $p$ " from the definitions of $H(E), V(E)$, and $O(E)$, we get, respectively, the sets $H_{1}(E)=H(E)+E, M(E)=V(E)+E=L(E)+E$, and $O_{1}(E)$ $=O(E)+E$. Evidently $H(E) \leqq V(E) \leqq O(E)$ and $H_{1}(E) \leqq M(E)$ $\leqq O_{1}(E)$. Since property $C$ means* that $I I(E) \equiv I(E)$, and since $M(E) \equiv C I C(E)$, a necessary and sufficient condition for property $\dagger C$ is that $M M(E) \equiv M(E)$. But in every space $\ddagger$

$$
H_{1} H_{1}(E) \equiv H_{1}(E) \text { and } O_{1} O_{1}(E) \equiv O_{1}(E) .
$$

Therefore if $M(E)=H_{1}(E)$ or $M(E)=O_{1}(E)$, property $C$ holds.

Theorem 2. If property $C$ holds, $H_{1}(E)=M(E)=O_{1}(E)$. Conversely, if any two of these three set functions coincide for all sets of the space, property $C$ holds.

From Theorem 2 we can deduce another necessary and sufficient condition for property $C$; namely, that some two of the set functions $H(E), V(E)$, and $O(E)$ coincide for all sets of the space.

4. The Borel-Lebesgue Property. Property $C$ holds if the space has the Borel property and every subset having that property is completely closed, or if the space has the Lindelöf property and every set having that property is completely closed; or if the space has the Borel-Lebesgue property and every set having that property is completely closed. If property $B$ is valid, every point is interior to a set having the property of Borel-Lebesgue, and every set having that property is completely closed, then

* Where $I(E)$ is the set of all interior points of $E$, and the symbol $\equiv$ means that the formula holds for every set $E$ of the space.

$\dagger$ This property is equivalent to the closure of derived sets in any space having the first three properties of Riesz, but the two properties are independent in $V$-spaces. If $M(Q)=Q$, we say that $Q$ is completely closed or $L$-closed.

$\ddagger$ These equalities are special cases of the relation $I_{I}(E)=I_{I}\left(E+I_{I}(E)\right)$ $>I_{I} I_{I}(E)$, where $I$ is now any set function and $I_{I}(E)$ is the set of points $p$ such that $I(W)>p$ implies $E \cdot I(W) \neq 0$. See Robinson, loc. cit., $\S 5$. If $E \cdot I(W)$ contains at least $\mu$ points, we say that $p<I_{I}^{\mu}(E)$. Then $I_{I}^{\mu}(E)>I_{I} I_{I}^{\mu}(E)$. From this we can prove that if property $C$ holds, the set of points $p$ such that every set to which $p$ is interior contains $\mu$ points of $E$, is a completely closed set. For $\mu=\mathrm{N}_{1}$, this means that points of condensation of any set $E$ form a completely closed set. 
a proof similar to that of Theorem 3 would show that property $C$ holds.

THEOREM 3. If for each point $p$ of a space $(P, K)$ there is a set $U_{p}$ to which $p$ is interior and such that every proper covering of $P$ is reducible to a finite proper covering of $U_{p}$, and if properties $B$ and $D$ hold, then property $C$ also holds.

Proof. We suppose $p$ is not an $M$-point of the set $E$ and prove it is not an $M$-point of $M(E)$. For each point $q$ of $M(E)$, let $V_{q}$ and $W_{q}$ be disjoined sets to which $p$ and $q$ are respectively interior. Then the $W_{q}$ 's together with $C(E)$ form a proper covering of $P$, which is reducible to a finite proper covering of $U_{p}$. Since $C(E)$ covers no points of $M(E), U_{p} \cdot M(E)$ is properly covered by sets $W_{q_{i}}$ which form a finite subfamily of the sets $W_{q}$. Therefore the product $U_{p_{i}} \cdot \Pi V_{q_{i}}$ to which $p$ is interior, contains no points of $M(E)$.

Consider a space consisting of the circumference of a circle. The limit points of a set $E$ are the points $p$ such that there are points of $E-p$ either (1) arbitrarily near $p$ in the direction clockwise from $p$, or (2) arbitrarily near $q$ (the point opposite $p$ ) in the direction counter clockwise from $q$. This space does not have property $C$. It satisfies properties $B$ and $D$ but not the other hypothesis of Theorem 3 . However, every proper covering is reducible to a finite simple covering.*

By a proof like that of Theorem 3 we can establish the following theorem.

Theorem 4. In a space $(P, K)$ having properties $B$ and $D$, any set $E$ such that every proper covering of $E$ is reducible to a finite simple one, is completely closed.

Theorems 5 and 6 which follow are corollaries of Theorem 4, while Theorem 7 is a consequence of a similar theorem in which

* In this Bulletin, vol. 37 (1931), p. 630, I stated erroneously that the above space is a Hausdorff space having the Borel-Lebesgue property. By a modification we can secure the space which we desired, a separable Hausdorff space having the Borel-Lebesgue property and satisfying the first but not the second enumerability axiom. Let the space consist of two concentric circles. Let the limit points of a set $E$ be as above, except that for any point $p$ on either circle, the point $q$ shall be the corresponding point on the other circle. In this example (and in one of $\$ 2$ ), we could just as well have taken two parallel closed segments, except that we desired to make the space homogeneous. 
the open set $B$ and $D$ properties are assumed and the set $E$ is only required to have the open set Borel-Lebesgue property.

Theorem 5. In a space $(P, K)$ having properties $B$ and $D, a$ necessary and sufficient condition that a set $E$ have the property of Borel-Lebesgue is that $E$ be L-closed and every infinite proper covering of $P$ be reducible to a proper covering of $E$ of lower power.

Theorem 6. In a space $P$ having properties $B$ and $D$, a necessary and sufficient condition that every infinite proper covering of a set $E$ be reducible to a finite simple one is that $E$ be L-closed and every infinite proper covering of $P$ be reducible to a finite simple covering of $E$.

THEOREM 7. In a space $P$ having the open set form of properties $B$ and $D$, a necessary and sufficient condition that every infinite family of open sets which covers $E$ be reducible is that $E$ be $L$ closed and every infinite family of open sets which covers $P$ be reducible to a covering of $E$ of lower power.

Each of the reducibility properties of Theorems 3, 4, and 5 is equivalent to a nuclearity property and to a property relating to the closure of decreasing sequences of sets.* If these nuclearity and closure properties are substituted for the reducibility properties in Theorems 3, 4, and 5, additional theorems are secured. For example, in a space $(P, K)$ having properties $B$ and $D$, a necessary and sufficient condition that every infinite subset of the set $E$ have a proper nuclear point in $E$ is that $E$ be $L$-closed and every infinite subset of $E$ have a proper nuclear point in the space.

If the space satisfies the first enumerability axiom, the reducibility of enumerably infinite coverings can be substituted in Theorems 3-7 for the reducibility of coverings of any infinite power.

Princeton University

* See Robinson, loc. cit. 